\title{
Ultra-Lightweight Cement
}

\author{
Eighth Quarterly Technical Progress Report
}

July 1 to September 30, 2002

Fred Sabins

Issued October 31, 2002

DOE Award Number

DE-FC26-00NT40919

Submitted by Cementing Solutions, Inc.

4613 Brookwoods Drive

Houston, TX 77092 


\section{Disclaimer}

This report was prepared as an account of work sponsored by an agency of the United States Government. Neither the United States Government nor any agency thereof, nor any of their employees, makes any warranty, express or implied, or assumes any legal liability or responsibility for the accuracy, completeness, or usefulness of any information, apparatus, product, or process disclosed, or represents that its use would not infringe privately owned rights. Reference herein to any specific commercial product, process, or service by trade name, trademark, manufacturer, or otherwise does not necessarily constitute or imply its endorsement, recommendation, or favoring by the United States Government or any agency thereof. The views and opinions of authors expressed herein do not necessarily state or reflect those of the United States Government or any agency thereof. 


\section{Abstract}

The objective of this project is to develop an improved ultra-lightweight cement using ultra-lightweight hollow glass spheres (ULHS). This report includes results from laboratory testing of ULHS systems along with other lightweight cement systems, including foamed and sodium silicate slurries. During this project quarter, a comparison study of the three cement systems examined the effect that cement drillout has on the three cement systems. Testing to determine the effect of pressure cycling on the shear bond properties of the cement systems was also conducted. This report discusses testing that will be performed for analyzing the alkali-silica reactivity of ULHS in cement slurries, as well as the results of Field Tests 1 and 2. 


\section{Table of Contents}

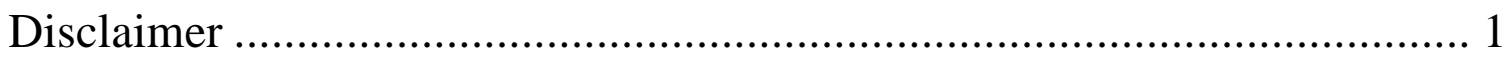

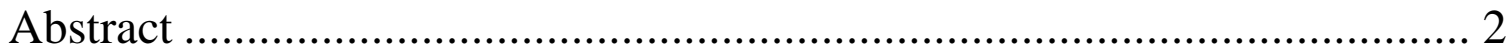

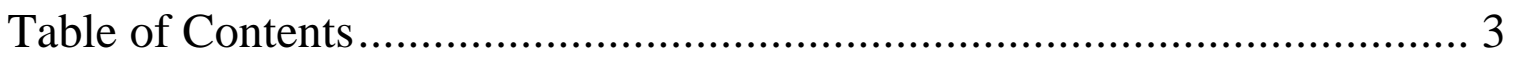

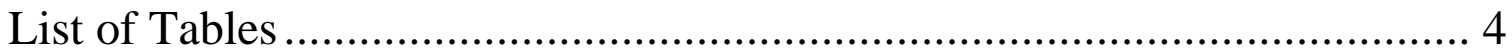

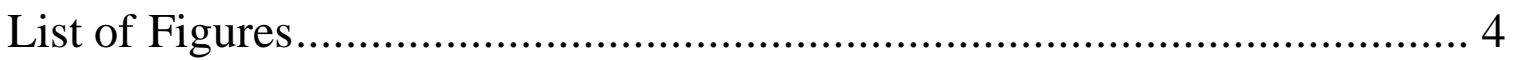

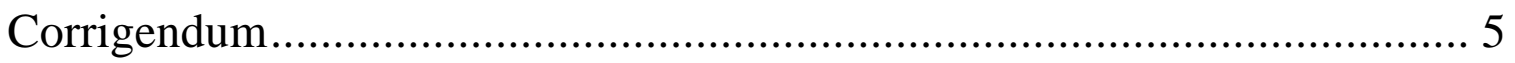

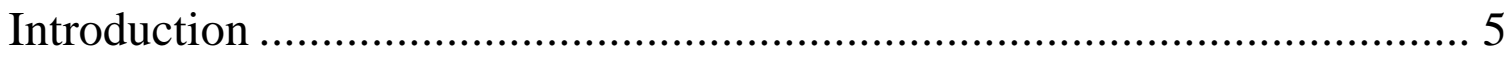

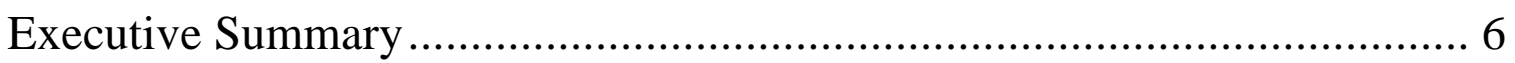

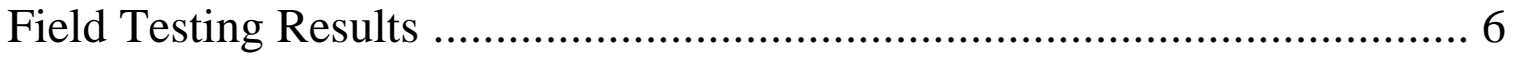

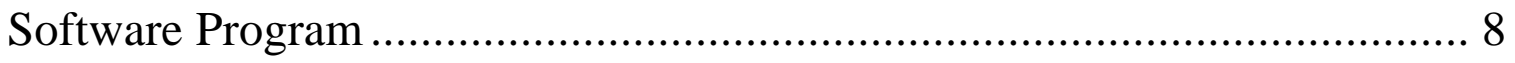

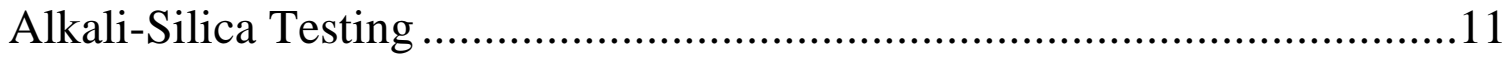

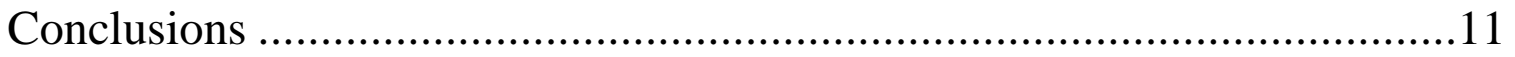

List of Acronyms and Abbreviations......................................................12

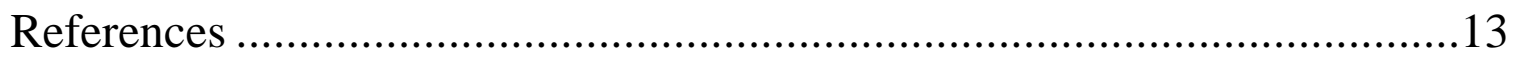

Appendix A, Quality Control Testing ..................................................A-1

Appendix B, On-Site Testing Summary of DOE Field Job \#2 ..................... B-1

Appendix C, Halliburton Cement Test Report ............................................

Appendix D, Preliminary Halliburton Job Recommendations .......................D-1

Appendix E, Alkali-Silica Reactivity (ASR) Testing ..................................E-1 


\section{List of Tables}

Table 1—Field Test 2 Parameters and Results

\section{List of Figures}

Figure $1-\mathrm{CBL}$ and ultrasonic log for $10 \mathrm{lb} / \mathrm{gal}$ ULHS slurry

Figure 2-General well information

Figure 3-Lead cement parameters

Figure 4-Tail cement parameters

Figure 5-Output screen containing job design requirements 


\section{Corrigendum}

One of the cement types used in the project's tests has been inaccurately referred to as Class A in previous quarterly progress reports. The cement is actually Type I cement. Although Class A and Type I cements are very similar, they are different cements.

In previous progress reports, the "Class A" designation should be replaced with "Type I" when used to describe the cement being tested.

\section{Introduction}

Oilwell cementing involves placing a pumpable slurry of Portland cement, additives, and water into a wellbore. The slurry is pumped into the annular space between the borehole and a steel pipe (called a casing) that acts as a conduit from the reservoir to the surface. The setting of cement in place serves three important functions: (1) it supports the casing in the hole, (2) it isolates various formations from one another, and (3) it controls fluid movement within the well.

Typically, cement fluid density is anywhere from 12 to $17 \mathrm{lb} / \mathrm{gal}$. Certain conditions that require the application of low-density cements can be encountered during the well construction process. Lower density is required (1) to limit hydrostatic pressure on the formation, and (2) to prevent the formation from fracturing and imbibing the well fluid. This phenomenon, known as lost circulation, increases drilling and completion times and increases construction cost because of the need for expensive remedial treatments. Lost circulation most commonly occurs in the upper sections of the well, where surface and intermediate casings are installed. Because formations covered by these casings are relatively close to the Earth's surface, application temperatures for these low-density cements are low.

The minimum density achievable with conventional cements and additives is approximately $11 \mathrm{lb} /$ gal. At this density, the slurry's stability and set cement's strength and permeability are only marginally acceptable. Adding water to reduce the density of these conventional cements is impractical because additional water dilutes the cement, causing low strength and high permeability. Low temperatures, such as those in the upper well sections, delay strength development. To obtain a lower cement density or greater cement strength, ultra-lightweight materials must be mixed into the slurry.

Ultra-lightweight hollow spheres (ULHS) are excellent candidate materials for producing ultra-lightweight cements. These small hollow glass beads effectively trap air in the slurry, thereby lowering the slurry density without the addition of water.

This project is designed to develop cementing systems using ULHS through a carefully designed program of modeling, design, laboratory testing, and field testing. 


\section{Executive Summary}

Laboratory testing during the eighth quarter was a continuation and extension of testing conducted previously. Tests were performed for comparing the performance of slurries containing ULHS to that of foamed and sodium silicate cement systems. Comparison studies examined shear bond strength after pressure-cycling conditions.

Two field tests were designed and conducted to test the slurry's performance in actual formations. The first field test was designed to ensure that the slurry could be easily blended, mixed, and pumped on location, in actual wells with little trouble. The second field test was designed to test the slurry's performance in a land-based well that closely resembled deepwater operations.

This report provides the following information:

- Field testing results

- Cement Quality -Control Program and QC program data (Appendix A)

- Description of the completed cement slurry software program used to calculate the amount of ULHS needed for specific densities

- Alkali-Silica Reactivity Testing description and procedures (Appendix E)

- On-Site Testing Summary of DOE Field Job 2 (Appendix B) and the Cement Testing Report for Field Job 2 (Appendix C)

- Preliminary Halliburton Job Recommendations (Appendix D)

\section{Field Testing Result}

The second field test was performed in the Rocky Mountains in a well operated by the DOE and Rocky Mountain Oilfield Testing Center (RMOTC) in Wyoming. The second field test well conditions were 5,765 ft TD, with a static temperature of $200^{\circ} \mathrm{F}$ and a bottomhole circulating temperature of $130^{\circ} \mathrm{F}$. The previous well had been cemented with foam cement. These wells typically have problems with lost circulation and require highstrength cement and good zone isolation. This well required one hundred barrels of the ultra-lightweight cement slurry (using 3M 6K ULHS beads). The slurry was mixed and pumped with no problems, and the ULHS beads showed no breakage after one hour of conditioning at the surface. Complete fluid returns were observed during the entire cementing job. This was verified through density measurements every ten minutes. Tables 1 through 4 in Appendix B provide collected data.

Ultrasonic logs performed on the well after the cement operation showed good bonding to both pipe and formation (Fig. 1). In Figure 1, the callout entitled Good Pipe Bond shows that the amplitude of the ring of the pipe was very low indicating good coupling of the pipe. The second callout, Good Formation Bond, provides an estimation of the quality of the cement bond to the formation. In this case, the variable formation signal indicates a good cement formation bond. The third callout, High Impedence, High Compressive Strength, Good Bond, indicates that the impedence values of the cement measured were high as evidenced by the dark color (the darker the color, the higher the cement strength). 


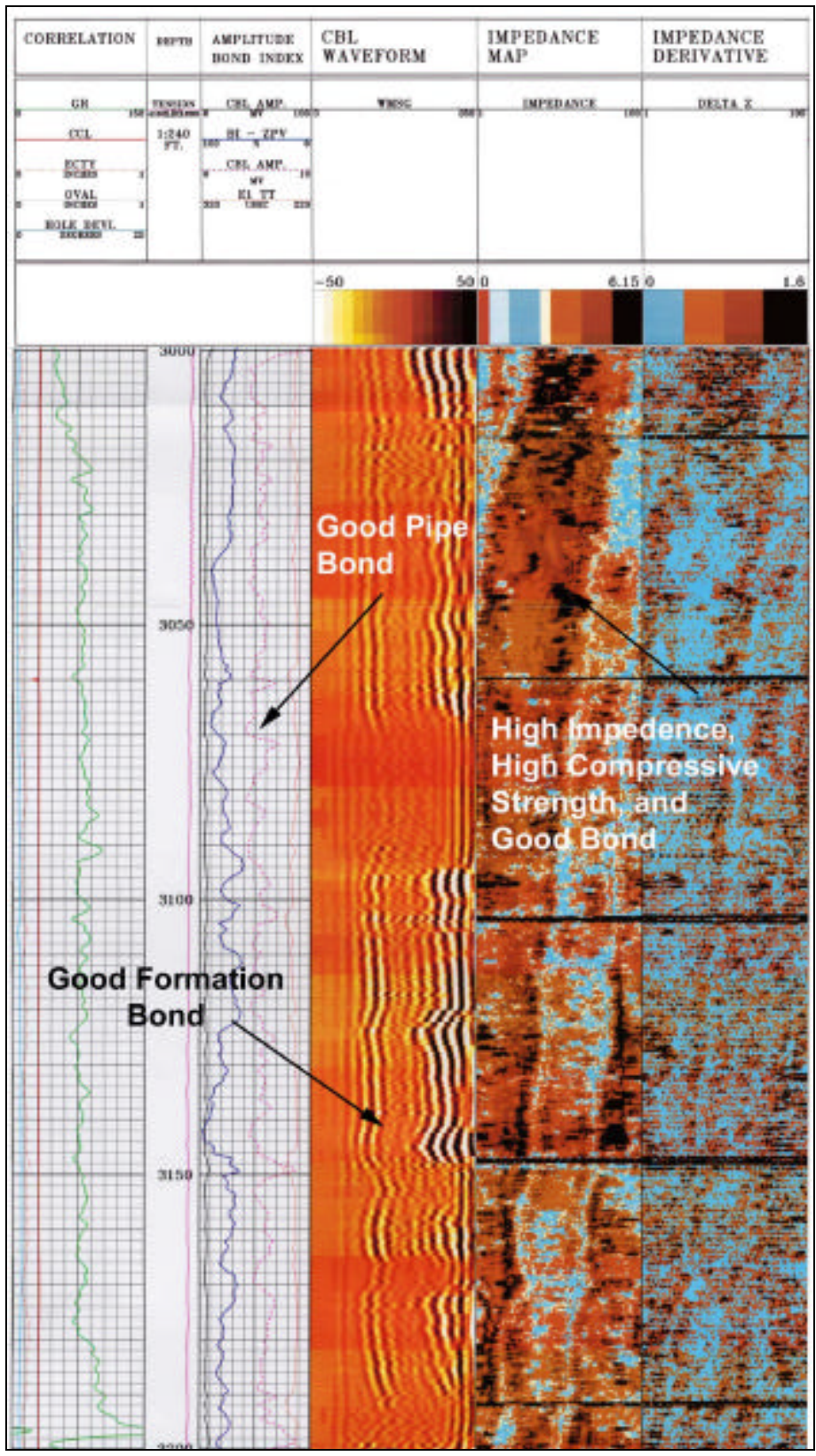

Figure 1-CBL and ultrasonic log for $10 \mathrm{lb} /$ gal ULHS slurry 
A summary of the field test parameters and results for Field Test 2 is shown in Table 1.

\begin{tabular}{|l|l|}
\hline \multicolumn{2}{|c|}{ Table 1-Field Test 2 Parameters and Results } \\
\hline County & Natrona County, Wyoming \\
\hline TD & $5,765 \mathrm{ft}$ \\
\hline Hole Size & $83 / 4 \mathrm{in}$. \\
\hline Production Casing & $7 \mathrm{in}$. \\
\hline Slurry Density & $10 \mathrm{lb} / \mathrm{gal}$ \\
\hline Lead Cement & $\mathrm{ULHS}$ w/ TXI Lighweight \\
\hline Tail Cement & TXI Lightweight \\
\hline Static Temperature & $200^{\circ} \mathrm{F}$ \\
\hline Bottomhole Circulating Temperature & $130^{\circ} \mathrm{F}$ \\
\hline
\end{tabular}

\section{Software Program}

To assist in designing cement slurries made with ULHS, a software program was developed to calculate the amount of ULHS beads required for specific densities. The program does not simulate cement jobs and fluid dynamics. Instead, the program focuses on the static final placement of the fluids (i.e., the slurry composition and slurry properties such as yield and density).

The software program uses an inputted water requirement to calculate the amount of beads needed in the slurry.

The program has been distributed to the project participants. Fig. 2 through Fig. 5 show the screens included in the program interface. 


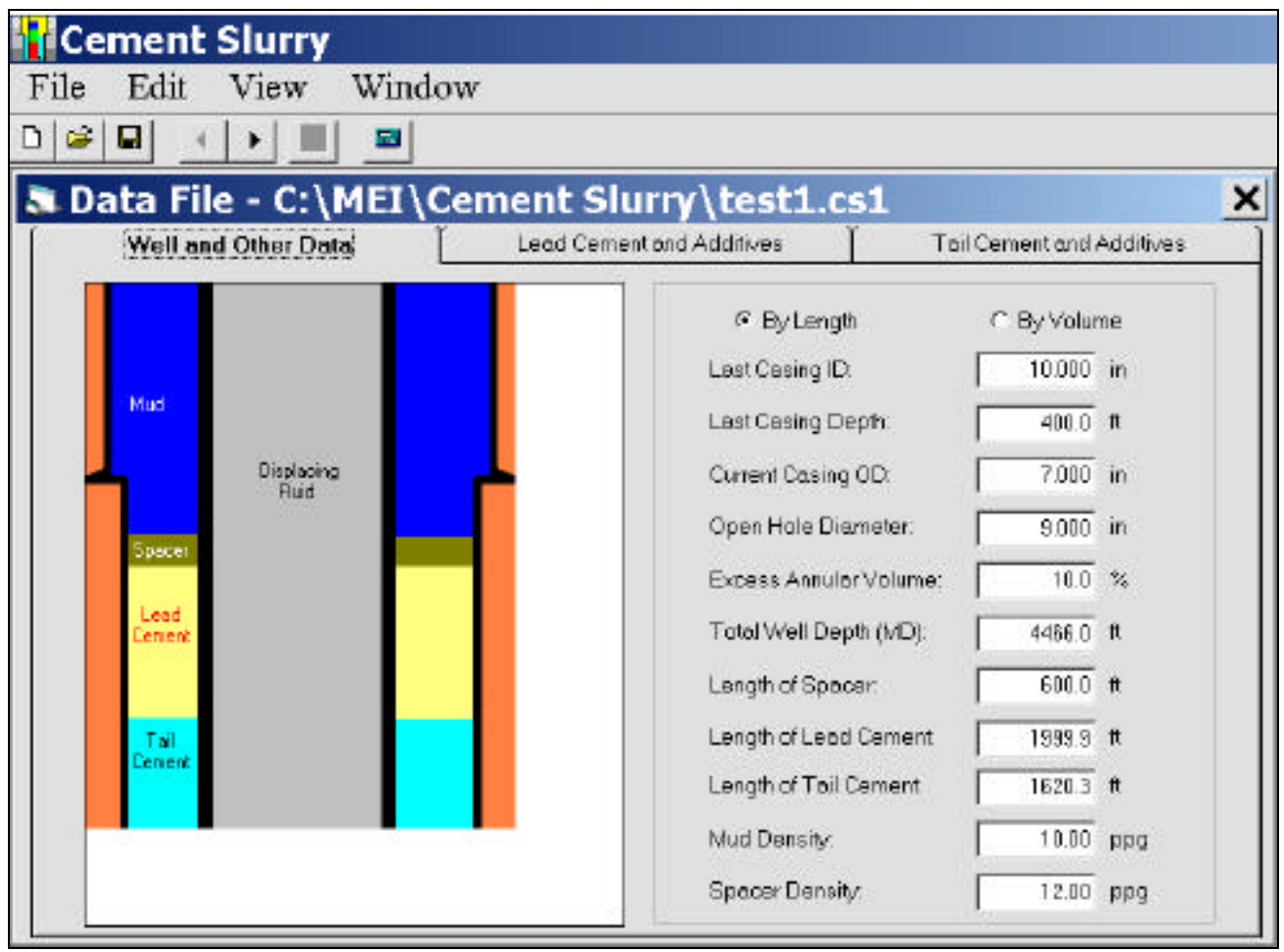

Figure 2-General well information

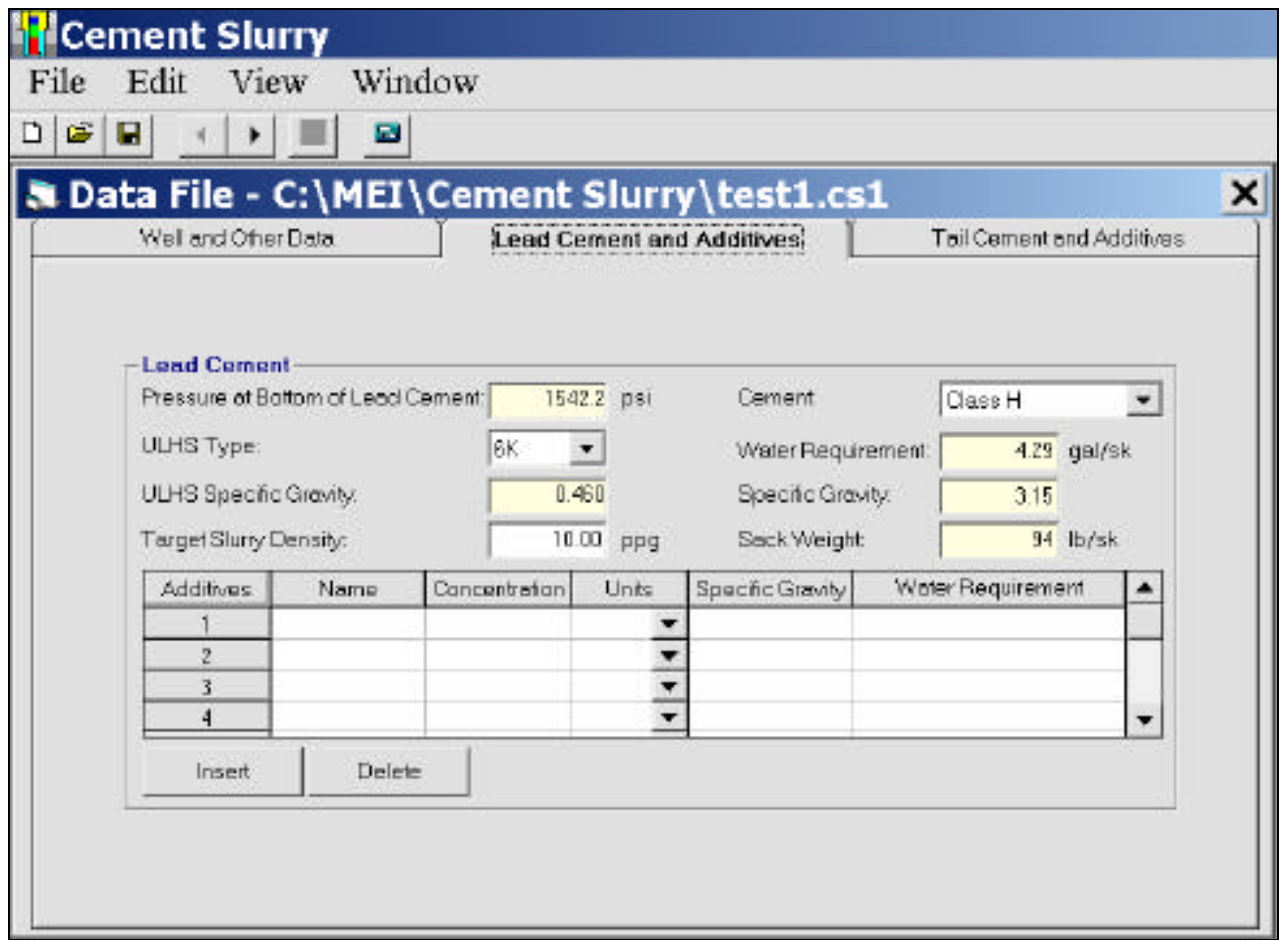

Figure 3-Lead cement parameters 


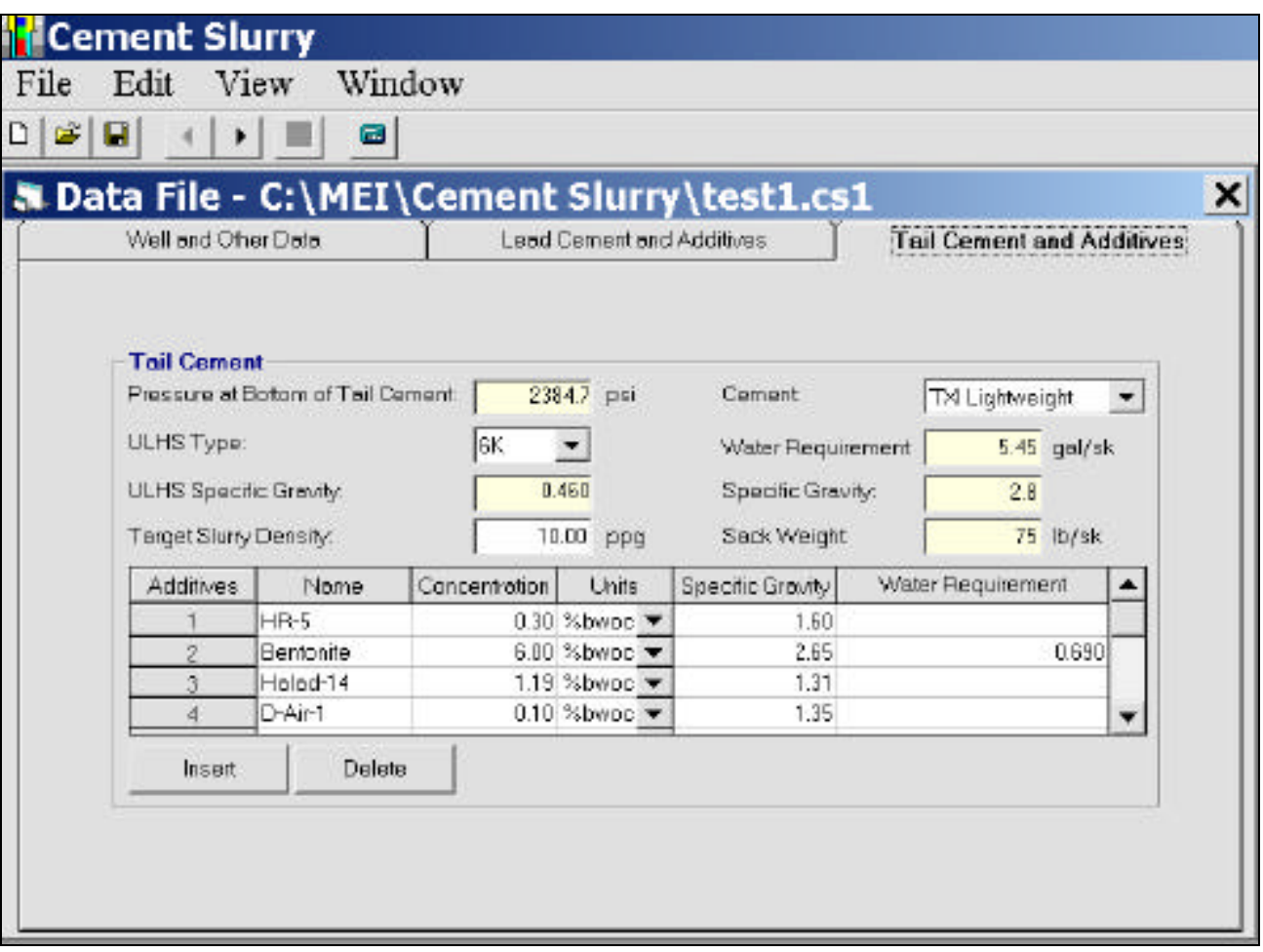

Figure 4-Tail cement parameters

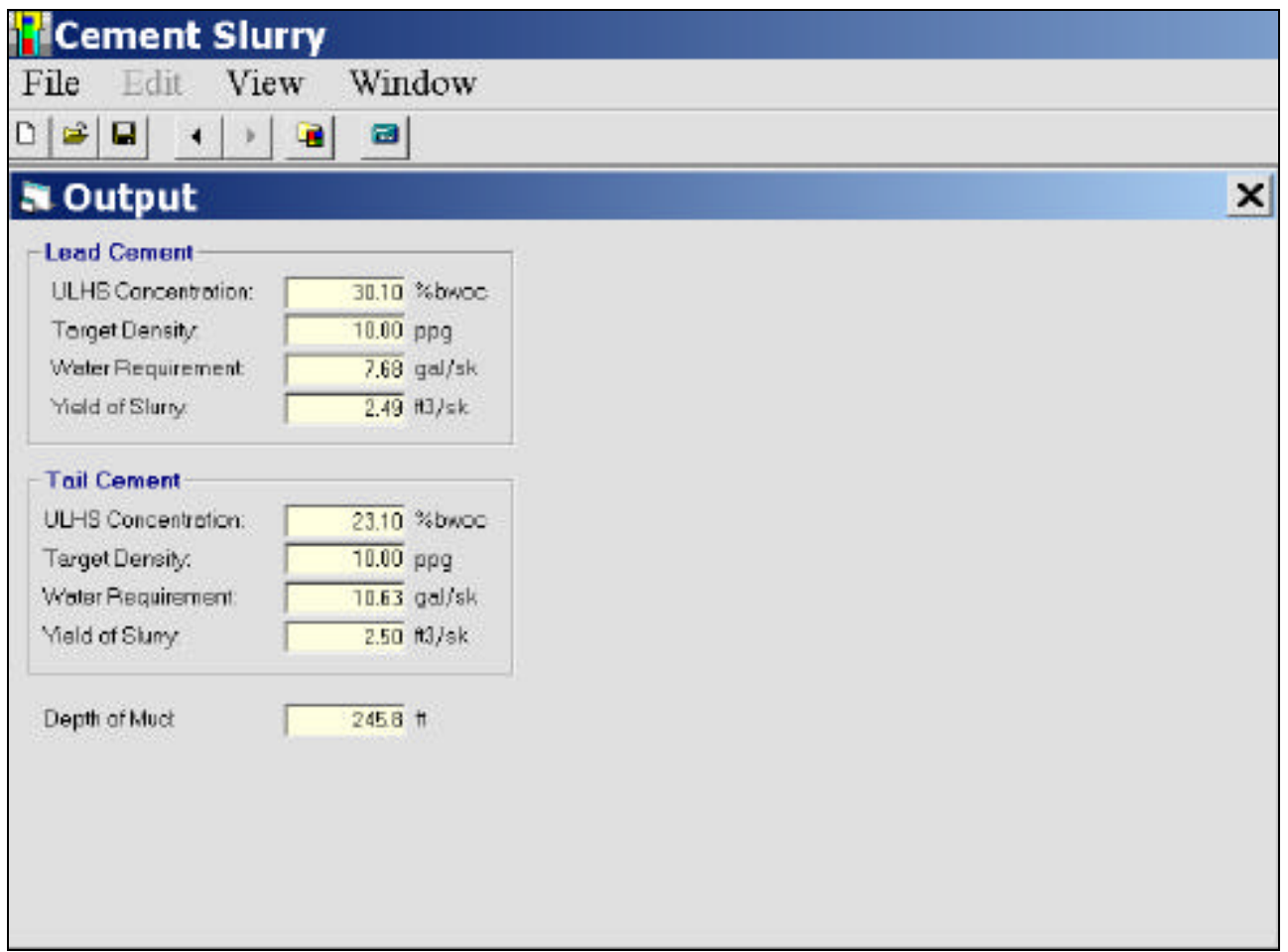

Figure 5-Output screen containing job design requirements 


\section{Alkali-Silica Testing}

Approval was made for a continuation of the current cooperative agreement with DOE. This agreement consisted of a new task to study the long-term effects of the Alkali-Silica Reaction (ASR) in ULHS cements. The goal of this task is to determine procedures and methods from the construction industry that are applicable to oil well cements, and conduct long-term tests to verify if ASR is occurring and when and how it manifests itself. Appendix D provides the proposed testing program.

\section{Conclusions}

1. A field job was successfully completed using the ULHS in Natrona County, Wyoming.

2. Ultrasonic and sonic logs performed on the well after the cement operation showed good cement bond to pipe, good cement bond to formation, and high compressive strengths.

3. A computer program was developed to assist in slurry design of ULHS cement systems.

4. A recommended procedure and testing protocol was developed for Alkali-Silica Reactivity of ULHS slurries. 


\title{
List of Acronyms and Abbreviations
}

\author{
API-American Petroleum Institute \\ ASR — alkali-silica reactivity \\ ASTM-American Society for Testing and Materials \\ $\mathrm{Bc}$-Bearden units of consistency \\ BHCT — bottomhole circulating temperature \\ BHST - bottomhole static temperature \\ BWOC - by weight of cement \\ $\mathrm{CaCl}_{2}$ - chemical formula for calcium chloride \\ $\mathrm{cp}-$ centipoise \\ gal-gallon \\ $\mathrm{H}_{2} \mathrm{O}$ - chemical formula for water \\ $\mathrm{hr}$-hour \\ ID-inner diameter \\ in.-inch \\ J-Joule \\ $\mathrm{lb}$ - pound \\ md-millidarcy \\ min-minute \\ OD-outer diameter \\ psi-pound per square inch \\ rev-revolution \\ rpm-revolutions per minute \\ $\mathrm{s}$ - second \\ sk-sack of cement \\ QC-quality control \\ TXI-Texas Industries \\ TXI LW - manufactured lightweight cement available from TXI \\ ULHS - ultra-lightweight hollow (glass) spheres \\ $3 \mathrm{~K}-3,000$-psi designation \\ $6 \mathrm{~K}-6,000-\mathrm{psi}$ designation
}




\section{References}

1. API Recommended Practice 10B: "Recommended Practices for Testing Well

Cements," 22nd Edition, American Petroleum Institute, Washington, D.C., December 1997. 


\section{Appendix A-Quality Control Testing}

\section{Class A}

\begin{tabular}{|c|c|c|c|c|c|c|c|c|c|c|c|}
\hline \multicolumn{2}{|c|}{ TXI Provided Information } & \multicolumn{3}{|c|}{ CSI Information } & \multicolumn{7}{|c|}{ CSI Thickening Time } \\
\hline $\mathrm{Cmt}$ & Mill Production & Date & $\mathrm{CSI}$ & Bucket & Test & $\%$ & Sch & Int & $15^{\prime}-30^{\prime}$ & 100 & Pass/ \\
\hline$\underline{\text { Class }}$ & Run Date & Received & $\underline{\log \#}$ & Opened & Date & $\underline{\mathrm{H}_{2} \mathrm{O}}$ & \# & $\underline{\mathrm{Bc}}$ & $\underline{\mathrm{Bc}}$ & $\underline{\mathrm{Bc}}$ & Fail \\
\hline Type I & $9 / 25$ to $9 / 27 / 00$ & $11 / 06 / 00$ & C-113 A-1 & $11 / 09 / 00$ & $11 / 15 / 00$ & 46.0 & 4 & 9 & 10 & $2: 17$ & Pass \\
\hline Type I & $9 / 25$ to $9 / 27 / 00$ & $11 / 06 / 00$ & C-113 A-2 & $11 / 20 / 00$ & $01 / 05 / 01$ & 46.0 & 4 & 8 & 11 & $2: 21$ & Pass \\
\hline Type I & $9 / 25$ to $9 / 27 / 00$ & $11 / 06 / 00$ & C-113 A-3 & $12 / 04 / 00$ & $01 / 09 / 01$ & 46.0 & 4 & 4 & ran & out & of sample \\
\hline Type I & $9 / 25$ to $9 / 27 / 00$ & $11 / 06 / 00$ & $C-113 A-4$ & $01 / 09 / 01$ & $01 / 17 / 01$ & 46.0 & 4 & 4 & 6 & $2: 09$ & Pass \\
\hline Type I & $9 / 25$ to $9 / 27 / 00$ & $11 / 06 / 00$ & C-189-A-8 & $07 / 11 / 01$ & $08 / 15 / 01$ & 46.0 & 4 & 6 & 8 & $1: 50$ & Pass \\
\hline Type I & $9 / 25-9 / 27 / 00$ & $11 / 06 / 00$ & C-189-A-17 & $08 / 30 / 01$ & $09 / 12 / 01$ & 46.0 & 4 & 6 & 8 & $2: 00$ & Pass \\
\hline Type I & $9 / 25-9 / 27 / 00$ & $11 / 06 / 00$ & C-189-A-6 & $10 / 16 / 01$ & $10 / 16 / 01$ & 46.0 & 4 & 7 & 11 & $1: 41$ & Pass \\
\hline Type I & $9 / 25-9 / 27 / 00$ & $11 / 06 / 00$ & C-189-A-6 & $10 / 16 / 01$ & $11 / 13 / 01$ & 46.0 & 4 & 7 & 13 & $1: 53$ & Pass \\
\hline Type I & $9 / 25-9 / 27 / 00$ & $11 / 06 / 00$ & C-189-A-6 & $10 / 16 / 01$ & $12 / 21 / 01$ & 46.0 & 4 & 6 & 11 & $1: 46$ & Pass \\
\hline Type I & $9 / 25-9 / 27 / 00$ & $11 / 06 / 00$ & C-189-A-11 & $01 / 17 / 02$ & $01 / 14 / 02$ & 46.0 & 4 & 8 & 15 & $1: 42$ & Pass \\
\hline Type I & $9 / 25-9 / 27 / 00$ & $11 / 06 / 00$ & C-189-A-12 & $02 / 17 / 02$ & $02 / 11 / 02$ & 46.0 & 4 & 15 & 17 & $1: 40$ & Pass \\
\hline Type I & $9 / 25-9 / 27 / 00$ & $11 / 06 / 00$ & C-189-A-13 & $02 / 28 / 02$ & $03 / 04 / 02$ & 46.0 & 4 & 14 & 12 & $2: 10$ & Pass \\
\hline Type I & $9 / 25-9 / 27 / 00$ & $11 / 06 / 00$ & C-189-A-14 & $03 / 27 / 02$ & $04 / 02 / 02$ & 46.0 & 4 & 21 & 19 & $1: 46$ & Pass \\
\hline Type I & $4 / 19-4 / 21 / 02$ & $05 / 02 / 02$ & C-418-A & $05 / 02 / 02$ & $05 / 03 / 02$ & 46.0 & 4 & 27 & 28 & $2: 15$ & Pass \\
\hline Type I & $4 / 19-4 / 21 / 02$ & $05 / 02 / 02$ & C-418-B & $06 / 19 / 02$ & $06 / 25 / 02$ & 46.0 & 4 & 32 & 30 & $2: 00$ & Pass \\
\hline Type I & $4 / 19-4 / 21 / 02$ & $05 / 02 / 02$ & C-418-B & $06 / 19 / 02$ & $07 / 24 / 02$ & 46.0 & 4 & 29 & 28 & $1: 59$ & Pass \\
\hline Type I & $4 / 19-4 / 21 / 02$ & $05 / 02 / 02$ & C-418-B & $06 / 19 / 02$ & $08 / 08 / 02$ & 46.0 & 4 & 29 & 28 & $1: 57$ & Pass \\
\hline Type I & $4 / 19-4 / 21 / 02$ & $05 / 02 / 02$ & C-418-B & $06 / 19 / 02$ & $09 / 24 / 02$ & 46.0 & 4 & 29 & 28 & $1: 59$ & Pass \\
\hline
\end{tabular}

\section{Lightweight}

\begin{tabular}{|c|c|c|c|c|c|c|c|c|c|c|c|c|}
\hline \multicolumn{3}{|c|}{ TXI Provided Information } & \multicolumn{3}{|c|}{ CSI Information } & \multicolumn{7}{|c|}{ CSI Thickening Time } \\
\hline $\mathrm{Cmt}$ & Mill Production & & Date & $\mathrm{CSI}$ & Bucket & Test & $\%$ & Sch & Int & 70 & 100 & Pass/ \\
\hline$\underline{\text { Class }}$ & Run Date & $\underline{\text { Grind }}$ & $\underline{\text { Received }}$ & $\underline{\log \#}$ & Opened & Date & $\underline{\mathrm{H}_{2} \mathrm{O}}$ & $\#$ & $\underline{B c}$ & $\underline{\mathrm{Bc}}$ & $\underline{\mathrm{Bc}}$ & Fail \\
\hline LW & $9 / 15$ to $9 / 18 / 00$ & 62 & $11 / 06 / 00$ & C-108 B-1 & $11 / 07 / 00$ & $11 / 15 / 00$ & 75.0 & 5 & 6 & $2: 02$ & $2: 20$ & \\
\hline LW & $9 / 15$ to $9 / 18 / 00$ & 62 & $11 / 06 / 00$ & C-108 B-2 & $12 / 05 / 00$ & $01 / 05 / 01$ & 75.0 & 5 & 5 & $2: 00$ & 2:17 & \\
\hline LW & $9 / 15$ to $9 / 18 / 00$ & 62 & $11 / 06 / 00$ & C-108-B-12 & $07 / 03 / 01$ & $08 / 14 / 01$ & 75.0 & 5 & 7 & $2: 03$ & 2:22 & \\
\hline LW & $9 / 15$ to $9 / 18 / 00$ & 62 & $11 / 06 / 00$ & C-108-B-7 & $09 / 05 / 01$ & 09/10/01 & 75.0 & 5 & 6 & $1: 57$ & 2:17 & \\
\hline LW & $9 / 15$ to $9 / 18 / 00$ & 62 & $11 / 06 / 00$ & C-108-B-14 & $09 / 24 / 01$ & $10 / 16 / 01$ & 75.0 & 5 & 4 & $2: 07$ & 2:26 & \\
\hline LW & $9 / 15$ to $9 / 18 / 00$ & 62 & $11 / 06 / 00$ & C-108-B-14 & $09 / 24 / 01$ & $11 / 13 / 01$ & 75.0 & 5 & 5 & $2: 05$ & 2:25 & \\
\hline LW & $9 / 15$ to $9 / 18 / 00$ & 62 & $11 / 06 / 00$ & C-108-B-14 & $09 / 24 / 01$ & $12 / 21 / 01$ & 75.0 & 5 & 7 & $2: 03$ & 2:22 & \\
\hline LW & $9 / 15$ to $9 / 18 / 00$ & 62 & $11 / 06 / 00$ & C-108-B-13 & $12 / 04 / 01$ & $01 / 11 / 02$ & 75.0 & 5 & 8 & $1: 58$ & 2:12 & \\
\hline LW & $9 / 15$ to $9 / 18 / 00$ & 62 & $11 / 06 / 00$ & C-108-B-15 & $01 / 22 / 02$ & $02 / 08 / 02$ & 75.0 & 5 & 7 & $2: 05$ & 2:15 & \\
\hline LW & $9 / 15$ to $9 / 18 / 00$ & 62 & $11 / 06 / 00$ & C-108-B-18 & $02 / 25 / 02$ & $03 / 04 / 02$ & 75.0 & 5 & 13 & $2: 11$ & $2: 27$ & \\
\hline LW & $9 / 15$ to $9 / 18 / 00$ & 62 & $11 / 06 / 00$ & C-108-B-18 & $02 / 25 / 02$ & $04 / 09 / 02$ & 75.0 & 5 & 23 & $1: 54$ & 2:16 & \\
\hline LW & $9 / 15$ to $9 / 18 / 00$ & 62 & $11 / 06 / 00$ & C-108-B-16 & $05 / 15 / 02$ & $05 / 16 / 02$ & 75.0 & 5 & 19 & $2: 00$ & 2:12 & \\
\hline LW & $9 / 15$ to $9 / 18 / 00$ & 62 & $11 / 06 / 00$ & C-108-B-18 & $02 / 25 / 02$ & $06 / 26 / 02$ & 75.0 & 5 & 24 & $1: 53$ & $2: 10$ & \\
\hline LW & $9 / 15$ to $9 / 18 / 00$ & 62 & $11 / 06 / 00$ & C-108-B-18 & $02 / 25 / 02$ & $07 / 26 / 02$ & 75.0 & 5 & 23 & $1: 59$ & 2:09 & \\
\hline LW & $9 / 15$ to $9 / 18 / 00$ & 62 & $11 / 06 / 00$ & C-108-B-18 & $02 / 25 / 02$ & $08 / 22 / 02$ & 75.0 & 5 & 23 & $2: 05$ & 2:15 & \\
\hline LW & $9 / 15$ to $9 / 18 / 00$ & 62 & $11 / 06 / 00$ & C-108-B-18 & $02 / 25 / 02$ & $09 / 12 / 02$ & 75.0 & 5 & 23 & $2: 00$ & $2: 10$ & \\
\hline
\end{tabular}




\section{Class $\mathrm{H}$}

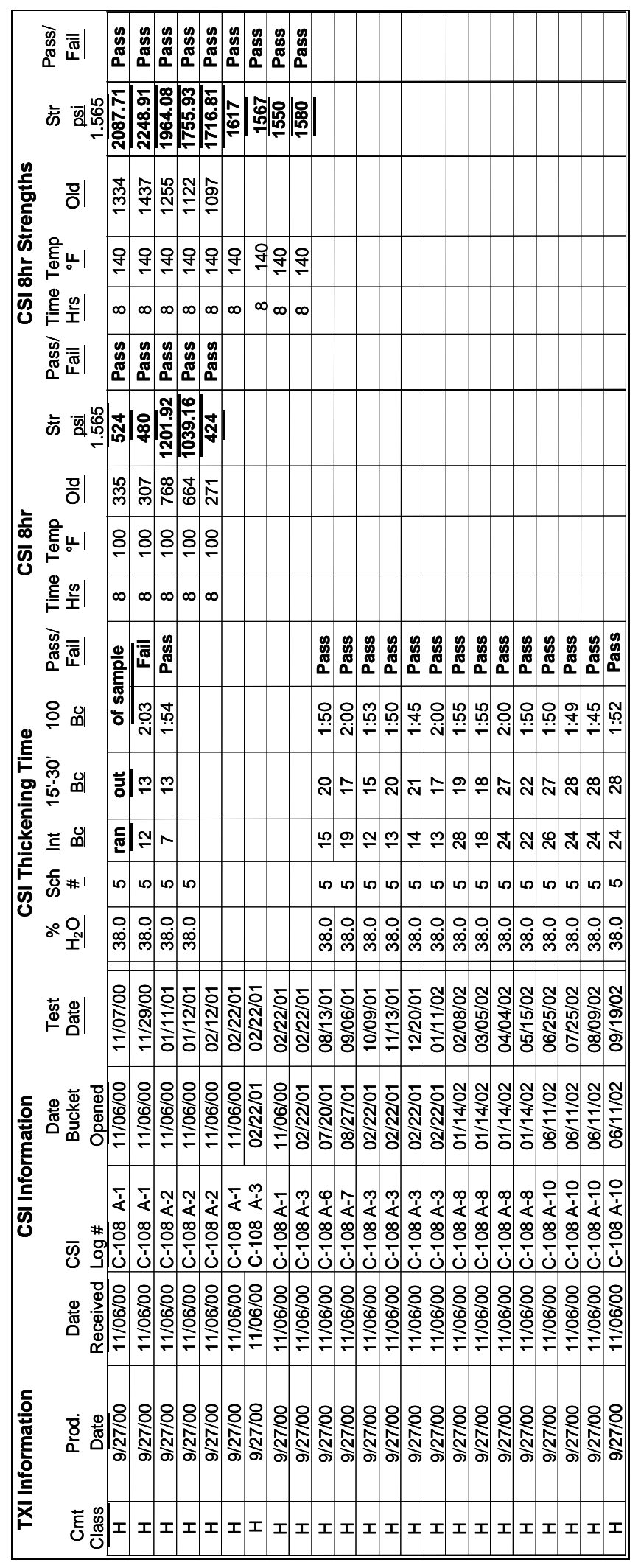




\section{Appendix B-On-Site Testing Summary of DOE Field Job \#2}

A 10 ppg, TXI Lightweight ULHS lead cement slurry was pumped as the lead slurry. The Tail slurry was a 13.5 ppg TXI Lightweight cement slurry mixed with $1 \%$ HALAD- 9. As seen in Table1, no changes in rheological properties occurred except for an increase in temperature after the slurry began to mix inside the RCM. The slurry was mixed on the $\mathrm{RCM}$ for an hour and rheologies were taken initially every five minutes; but after no change was noticed the sampling time was extended to every 10 minutes. The consistent rheologies indicated that no shearing of ULHS had occurred.

The rheologies shown in Tables 1 through 4 are much lower than the rheologies shown in Halliburton's lab report in Appendix C. We are uncertain of what caused this discrepancy.

Table 1.

\begin{tabular}{|c|c|c|c|c|c|c|c|c|c|c|c|c|}
\hline Lead & $\frac{X I \text { Lightweight }}{ \pm}$ & gent + & $\frac{25 \%}{\text { later }}$ & $\frac{\mathrm{JLHS}}{+0.20}$ & $\frac{+0 .}{\% \mathrm{CF}}$ & $\frac{\%}{R-3}$ & R-5 & +0.5 & $\% \mathrm{HA}$ & LAL & & \\
\hline$\underset{\text { (minutes) }}{\frac{\text { Time }}{\text { (minat }}}$ & $\frac{\text { Temperature }}{\left({ }^{\circ} \mathrm{F}\right)}$ & $\underline{300}$ & $\underline{200}$ & 100 & $\begin{array}{l}\text { Rhec } \\
\underline{60}\end{array}$ & $\begin{array}{r}\log \\
\underline{30} \\
\end{array}$ & $\begin{array}{l}\text { cal F } \\
\underline{20}\end{array}$ & $\begin{array}{c}\text { Prope } \\
\underline{10}\end{array}$ & $\begin{array}{c}\text { rties } \\
\underline{6}\end{array}$ & $\underline{3}$ & $\underline{\mathrm{PV}}$ & $\underline{\text { YP }}$ \\
\hline 0 & 91.7 & 86 & 60 & 34 & 26 & 18 & 14 & 12 & 10 & 10 & 78 & 8 \\
\hline 5 & 93.5 & 100 & 70 & 40 & 28 & 18 & 14 & 12 & 10 & 10 & 90 & 10 \\
\hline 15 & 93.5 & 96 & 64 & 38 & 26 & 18 & 16 & 10 & 10 & 10 & 87 & 9 \\
\hline 20 & 94.1 & 90 & 64 & 40 & 28 & 18 & 14 & 10 & 10 & 8 & 75 & 15 \\
\hline 30 & 94.5 & 92 & 62 & 36 & 24 & 16 & 14 & 10 & 10 & 8 & 84 & 8 \\
\hline 40 & 94.3 & 90 & 62 & 38 & 26 & 18 & 14 & 10 & 10 & 8 & 78 & 12 \\
\hline 50 & 94.2 & 82 & 60 & 36 & 26 & 18 & 14 & 10 & 10 & 8 & 69 & 13 \\
\hline 60 & 94.3 & 86 & 60 & 38 & 26 & 16 & 14 & 10 & 10 & 8 & 72 & 14 \\
\hline
\end{tabular}

Table 2 below lists the rheological properties of lead slurry sampled in the flow line as it was being pumped into the well. The slurry was pumped at a rate of $4 \mathrm{bpm}$. The slurries viscosity remained the same as those samples taken from the RCM through the first 10 minutes of sampling. 
Table 2

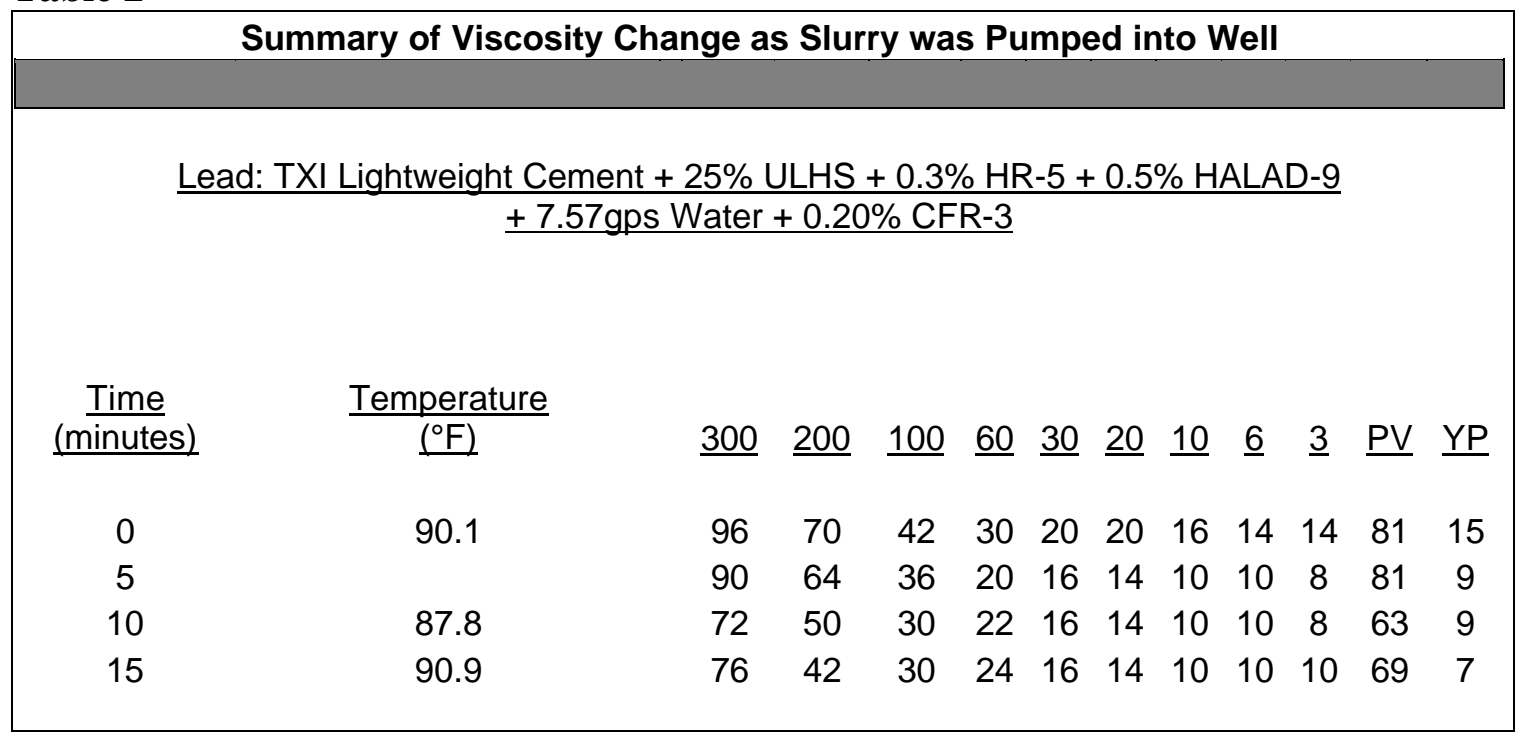

Pressurized Mud Balance was used during the job to verify density and record any changes that might occur during blending and agitation. Table 3 indicates time required to mix cement and achieve density of $10.0 \mathrm{ppg}$.

Table 3

\begin{tabular}{|cc|}
\hline Time & Density \\
$16: 15$ & $10.6 \mathrm{ppg}$ \\
$16: 25$ & $10.1 \mathrm{ppg}$ \\
$16: 40$ & $10.0 \mathrm{ppg}$ \\
\hline
\end{tabular}

Table 4 indicates time in blender agitating prior to pumping slurry downhole.

Table 4.

\begin{tabular}{|lc|}
\hline $16: 55$ & $9.9 \mathrm{ppg}$ \\
$17: 05$ & $10.0 \mathrm{ppg}$ \\
$17: 10$ & $10.0 \mathrm{ppg}$ \\
$17: 20$ & $10.0 \mathrm{ppg}$ \\
$17: 30$ & $10.0 \mathrm{ppg}$ \\
$17: 45$ & $10.0 \mathrm{ppg}$ \\
$17: 50$ & $10.0 \mathrm{ppg}$ \\
$18: 00$ & $10.0 \mathrm{ppg}$ \\
$18: 15$ & $10.0 \mathrm{ppg}$ \\
$18: 30$ & $10.0 \mathrm{ppg}$ \\
$18: 35$ & $10.0 \mathrm{ppg}$ \\
$18: 45$ & $10.0 \mathrm{ppg}$ \\
\hline
\end{tabular}

The cement remained in the blender for 1 hour and 50 minutes prior to pumping without any change in density. Aeration of slurry caused loss of prime to centrifugal pump twice 
during agitation period. This is a normal phenomenon when no additive is used to prevent foaming of slurry.

\section{Job Log}






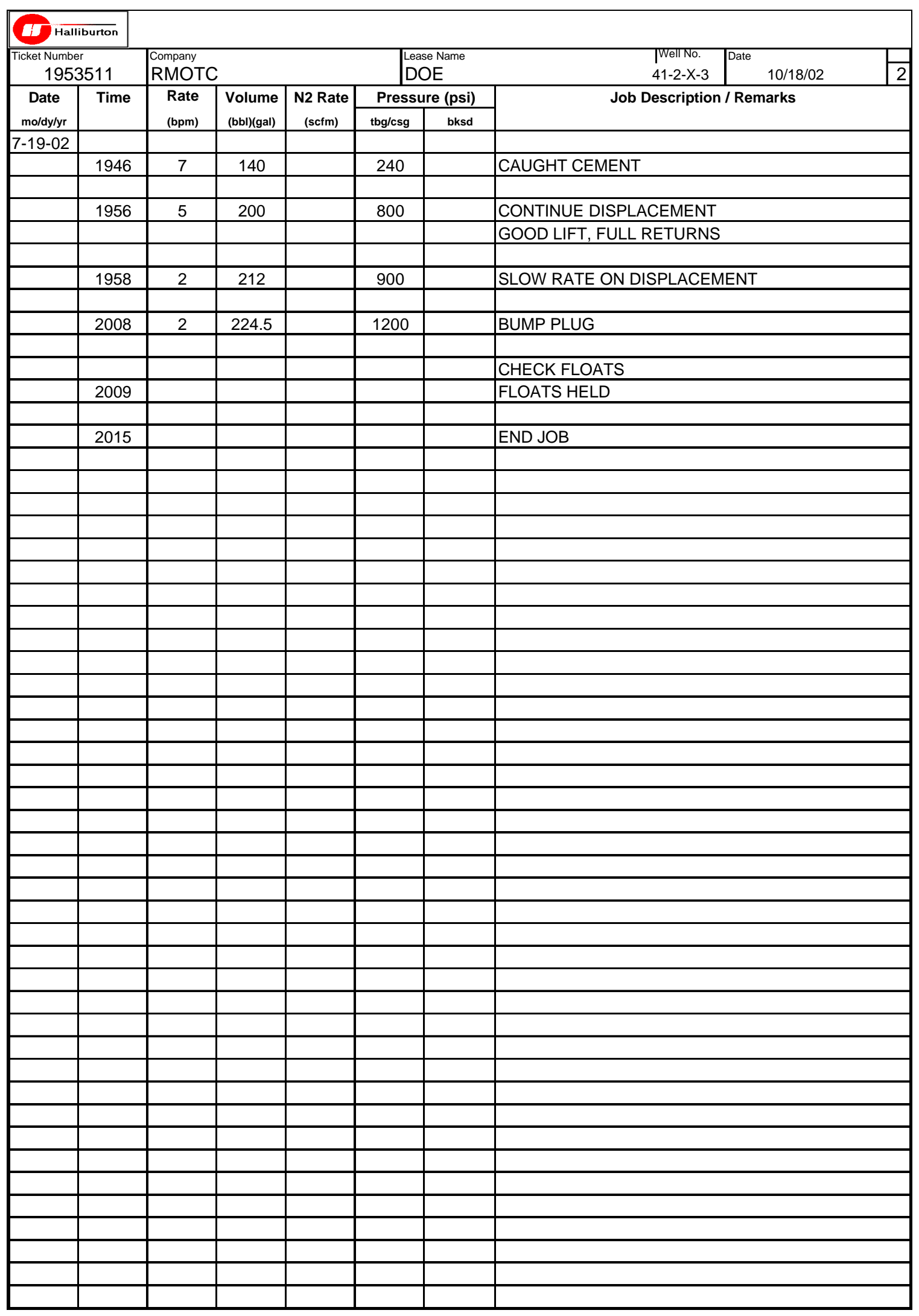




\section{Job Chart}

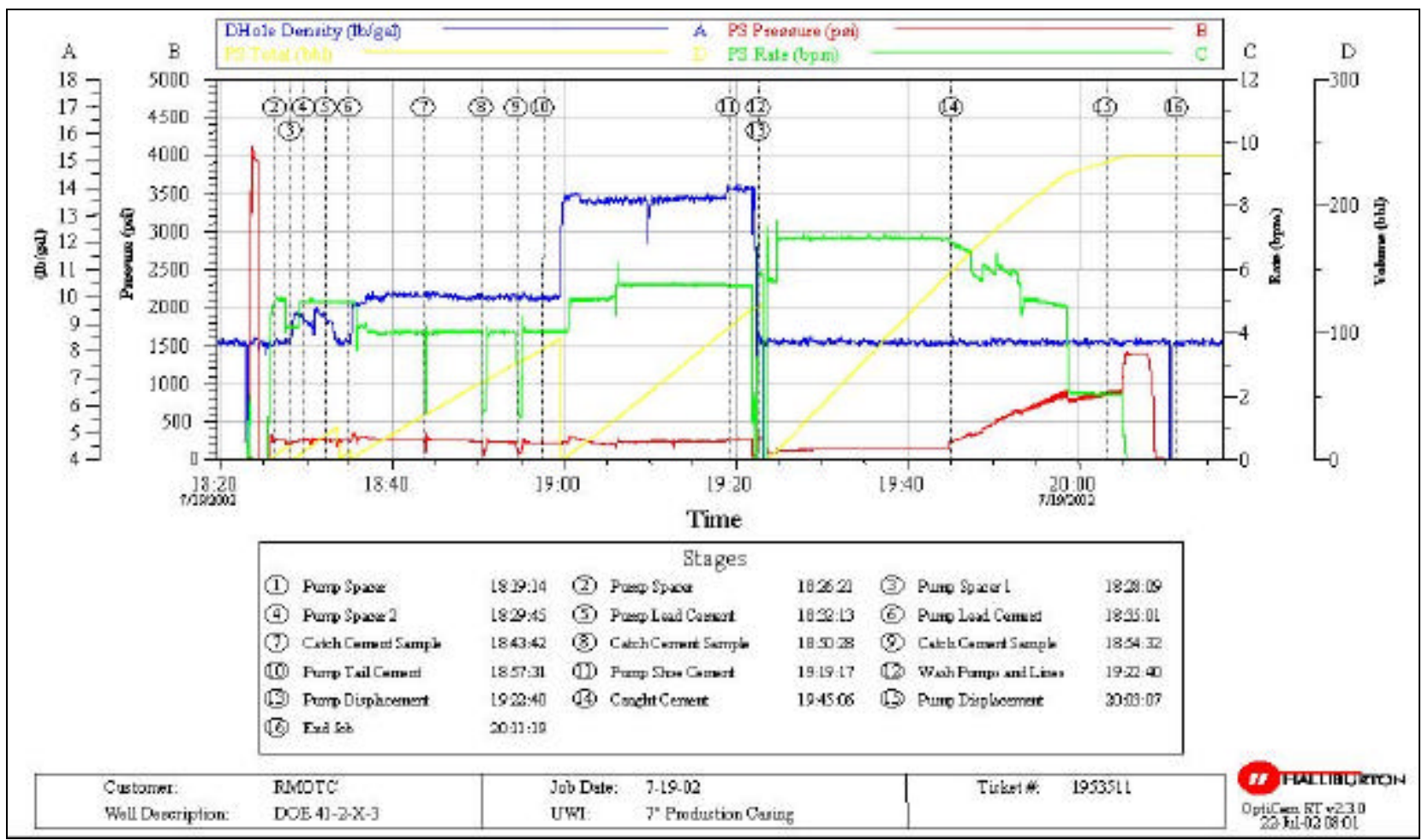




\title{
Appendix C-Halliburton Cement Test Report
}

\author{
-AL-IBUBTON \\ CEMENT TEST REPORT \\ HALLIBURTON ENERGY SERVICES \\ ROCKY MOUNTAIN NWA LABORATORY \\ EVANSVILLE, WY
}

Date: 19 Jul 02

Report: RMOTC/CSI, C02-0211

To: Brian Carty

- Halliburton Energy Services

- Casper, Wyoming

\section{PURPOSE}

The purpose of this work was to determine the thickening times and rheologies of the submitted slurry pilot designs and bulk plant blends using the conditions listed below.

\section{SCOPE AND PROCEDURE}

All tests were performed according to modified API Spec 10. Re: worksheet \# 1077, 1078, 1082, 1083

\section{WELL INFORMATION AND TESTING CONDITIONS}

Company
well Name
Job Type
District
State
Depth
BHST
BHCT

$\begin{array}{ll}- & \text { RMOTC/CSI } \\ - & \text { DOE 41-2-X-3 } \\ - & \text { Casing } \\ - & \text { Casper } \\ - & \text { Wyoming } \\ - & 5700 \text { feet } \\ - & 200 \text { degrees F } \\ - & 130 \text { degrees F } \\ - & 3500 \text { psi }\end{array}$

Respectfully submitted,

Nancy K. Lang

HALLIBURTON ENERGY SERVICES

CC: Casper Cementing 


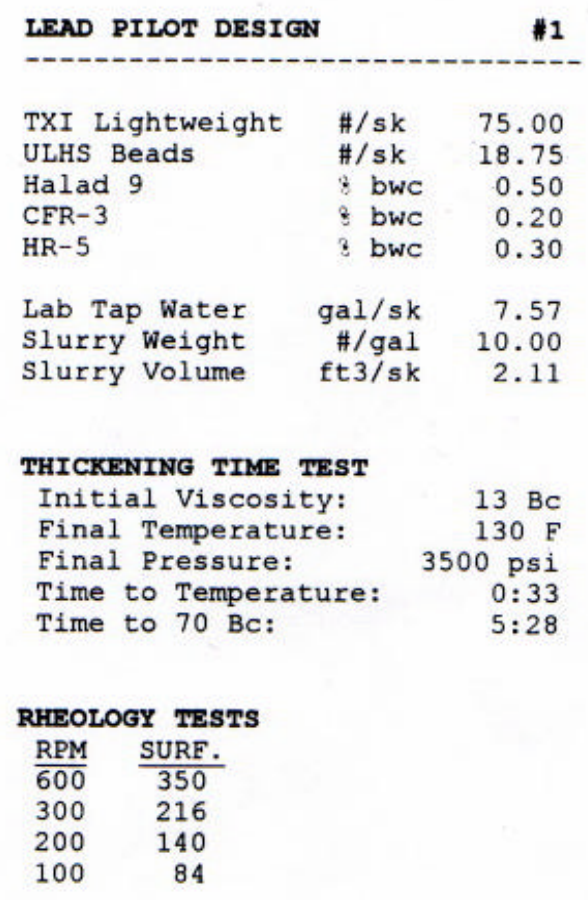

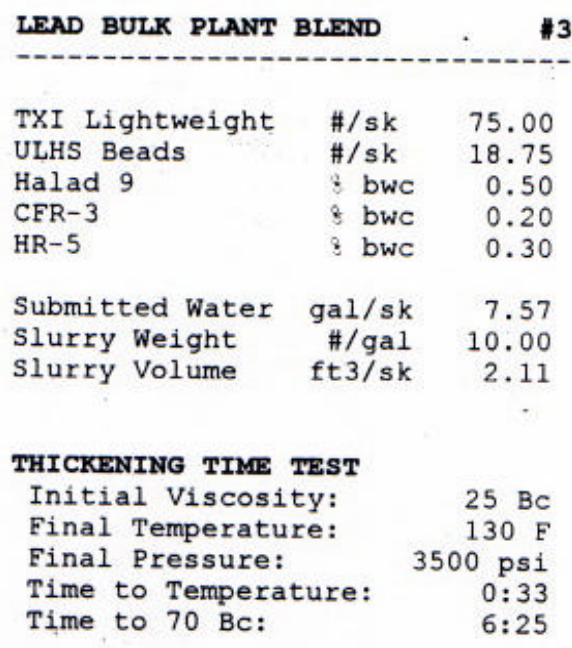

$\begin{aligned} & \text { RHEOLOG TESTS } \\ & \text { RPM }\end{aligned}$
$\begin{array}{llrr}\frac{\text { SURE. }}{600} & \frac{110 \mathrm{~F}}{360} & \frac{130 \mathrm{~F}}{370} & \frac{1366}{366} \\ 300 & 282 & 240 & 228 \\ 200 & 198 & 160 & 156 \\ 100 & 110 & 88 & 86\end{array}$


TAIL PILOT DESIGN

$\begin{array}{llr}\text { TXI Lightweight } & \begin{array}{l}\text { \#/sk } \\ \text { Halad 9 }\end{array} & 75.00 \\ & & \\ & & \\ & & \\ \text { Submitted Water } & \text { gal/sk } & 6.00 \\ \text { Slurry Weight } & \text { \#/gal } & 13.50 \\ \text { Slurry Volume } & \mathrm{ft} 3 / \mathrm{sk} & 1.25\end{array}$

\section{THICKENING TIME TEST}

Initial Viscosity:

Final Temperature:

Final Pressure:

Time to Temperature:

Time to $70 \mathrm{Bc}$ :

\section{RHEOLOGY TESTS}

RPM SURF.

$\frac{\mathrm{RPM}}{600} \quad \frac{\mathrm{SRF}}{214}$

$300 \quad 124$

$200 \quad 94$

$100 \quad 60$
TAIL BULK PLANT BLEND

\#4

$\begin{array}{llr} & & \\ \text { TXI Lightweight } & \text { \#/sk } & 75.00 \\ \text { Halad 9 } & 8 \text { bwc } & 1.00 \\ \text { CFR-3 } & \text { \& bwc } & 0.40 \\ & & \\ \text { Submitted Water } & \text { gal/sk } & 6.02 \\ \text { Slurry Weight } & \# / g a l & 13.50 \\ \text { Slurry Volume } & \text { ft3/sk } & 1.25\end{array}$

THICKFNING TIME TEST

Initial Viscosity:

Final Temperature:

Final Pressure:

Time to Temperature:

Time to $70 \mathrm{Bc}$ :

$4 \mathrm{BC}$

$130 \mathrm{~F}$

3500 psi

$0: 33$

$3: 39$

RHEOLOGY TESTS

\begin{tabular}{|c|c|c|c|}
\hline$\frac{\text { RPM }}{600}$ & $\frac{\text { SURF. }}{154}$ & $\frac{110 \mathrm{~F}}{138}$ & $130 \mathrm{E}$ \\
\hline 30 & 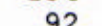 & & 120 \\
\hline & & 04 & 80 \\
\hline & & 36 & 6 \\
\hline
\end{tabular}

\footnotetext{
This report is based on sound engineering practices, but because of variable well conditions and other information which must be relied upon, Halliburton makes no warranty, express or $1 \mathrm{mplied}$, as to the accuracy of the data or of any calculations or opinions expressed herein. You agree that Halliburton shall not be liable for any loss or damage whether due to negligence or otherwise arising out of or in connection with such data, calculation or opinions.
} 


\section{Appendix D-Preliminary Halliburton Job Recommendations}

Appendix D contains the preliminary recommendation received from Halliburton. The well data and slurry composition shown in this appendix are different than what was actually performed on the job. Although this information is preliminary, it provides the details for volume calculations and critical well parameters. In addition, the well number shown below is wrong and should be DOE 41-2-X-3.

\section{HALLIBURTON}

Job Information

DOE 25-1-X-14

Surface Casing

Outer Diameter

Inner Diameter

Linear Weight

Casing Grade

Job Excess

Tensleep Production Hole Inner Diameter Job Excess

Tensleep Production Casing

Outer Diameter

Inner Diameter

Linear Weight

Casing Grade

Job Excess

Mud Weight

BHCT
0 - $725 \mathrm{ft}$ (MD)

9.625 in

8.921 in

$36 \mathrm{lb} / \mathrm{ft}$

J-55

$0 \%$

725 - $5900 \mathrm{ft}$ (MD)

10.540 in

$10 \%$

0 - $5900 \mathrm{ft}$ (MD)

7.000 in

6.366 in

$23 \mathrm{lb} / \mathrm{ft}$

J-55

$0 \%$

$0 \mathrm{lbm} / \mathrm{gal}$

0 degF 
Spacer:

$\begin{array}{ll}151.00 \mathrm{ft}^{*} 0.3387 \mathrm{ft}^{3} / \mathrm{ft}^{*} 10 \% & =56.25 \mathrm{ft}^{3} \\ \text { Total Spacer } & =56.15 \mathrm{ft}^{3} \\ & =10.00 \mathrm{bbl}\end{array}$

Spacer:

$\begin{array}{ll}301.00 \mathrm{ft}^{*} 0.3387 \mathrm{ft}^{3} / \mathrm{ft}^{*} 10 \% & =112.13 \mathrm{ft}^{3} \\ \text { Total Spacer } & =112.29 \mathrm{ft}^{3} \\ & =20.00 \mathrm{bbl}\end{array}$

Spacer:

$\begin{array}{ll}151.00 \mathrm{ft}^{*} 0.3387 \mathrm{ft}^{3} / \mathrm{ft}^{*} 10 \% & =56.25 \mathrm{ft}^{3} \\ \text { Total Spacer } & =56.15 \mathrm{ft}^{3} \\ & =10.00 \mathrm{bbl}\end{array}$

Cement : $(1515.00 \mathrm{ft}$ fill $)$ $1515.00 \mathrm{ft}^{*} 0.3387 \mathrm{ft}^{3} / \mathrm{ft}^{*} 10 \% \quad=564.37 \mathrm{ft}^{3}$

Total Lead Cement $\quad=564.37 \mathrm{ft}^{3}$

Sacks of Cement $\quad=270 \mathrm{sks}$

Cement : $(1748.00 \mathrm{ft}$ fill $)$ $1748.00 \mathrm{ft}^{*} 0.3387 \mathrm{ft}^{3} / \mathrm{ft}^{*} 10 \% \quad=651.17 \mathrm{ft}^{3}$ Tail Cement $\quad=651.17 \mathrm{ft}^{3}$

$=115.98 \mathrm{bbl}$

Shoe Joint Volume: $(44.00 \mathrm{ft}$ fill) $44.00 \mathrm{ft}^{*} 0.221 \mathrm{ft}^{3} / \mathrm{ft}$

$=9.73 \mathrm{ft}^{3}$

Tail plus shoe joint

$=1.73 \mathrm{bbl}$

$=660.90 \mathrm{ft}^{3}$

Total Tail

$=117.71 \mathrm{bbl}$

$=530 \mathrm{sks}$

Total Pipe Capacity:

$5900.00 \mathrm{ft}^{*} 0.221 \mathrm{ft}^{3} / \mathrm{ft} \quad=1304.11 \mathrm{ft}^{3}$

$=232.27 \mathrm{bbl}$

Displacement Volume to Shoe Joint: Capacity of Pipe - Shoe Joint

$=232.27 \mathrm{bbl}-1.73 \mathrm{bbl}$

$=230.54 \mathrm{bbl}$ 
Fluid Instructions

Fluid 1: Water Spacer

Water Spacer

Fluid 2: Spacer Sweep

Super Flush

$42 \mathrm{lbm} / \mathrm{bbl}$ Fresh Water (Base Fluid)

Fluid 3: Water Spacer

Water Spacer

Fluid 4: Lead Cement

TXI Lightweight

$75 \mathrm{lbm} / \mathrm{sk}$ TXI Lightweight (Cement-api)

$25 \% \quad$ Spherelite (Light Weight Additive)

$0.2 \%$ Halad(R)-9 (Low Fluid Loss Control)

Fluid 5: Tail Cement

TXI Lightweight

$75 \mathrm{lbm} / \mathrm{sk}$ TXI Lightweight (Cement-api)

$0.2 \% \quad$ Halad(R)-9 (Low Fluid Loss Control)
Fluid Density: $\quad 8.34 \mathrm{lbm} / \mathrm{gal}$

Fluid Volume: $10 \mathrm{bbl}$

Fluid Density: $\quad 9.20 \mathrm{lbm} / \mathrm{gal}$ Fluid Volume: $20 \mathrm{bbl}$

Fluid Density: $\quad \mathbf{8 . 3 4} \mathrm{lbm} / \mathrm{gal}$ Fluid Volume: $10 \mathrm{bbl}$

Fluid Weight $10 \mathrm{lbm} / \mathrm{gal}$

Slurry Yield: $\quad 2.09 \mathrm{ft}^{3} / \mathrm{sk}$

Total Mixing Fluid: $\quad 7.57 \mathrm{Gal} / \mathrm{sk}$

Top of Fluid: $\quad 2637 \mathrm{ft}$

Calculated Fill: $\quad 1515 \mathrm{ft}$

Volume: $\quad 100.51 \mathrm{bbl}$

Calculated Sacks: 270 sks

Proposed Sacks: 270 sks

Fluid 6: Water Spacer

Fresh Water Displacement

Fluid Density: $\quad \mathbf{8 . 3 4} \mathrm{lbm} / \mathrm{gal}$

Fluid Volume: $230.54 \mathrm{bbl}$

Fluid Weight $\quad 13.50 \mathrm{lbm} / \mathrm{gal}$

Slurry Yield: $\quad 1.25 \mathrm{ft}^{3} / \mathrm{sk}$

Total Mixing Fluid: $\quad 6.10 \mathrm{Gal} / \mathrm{sk}$

Top of Fluid: $\quad 4152 \mathrm{ft}$

Calculated Fill: $\quad 1748 \mathrm{ft}$

Volume: $\quad 117.71 \mathrm{bbl}$

Calculated Sacks: 530 sks

Proposed Sacks: 530 sks

The Cement and Hollow Beads will be Customer Supplied. 


\section{Appendix E-Alkali-Silica Reactivity (ASR) Testing}

\section{Objective}

The goals of this project are to study the long-term effects of the alkali-silica reaction in cements and to conduct a comprehensive evaluation of the ULHS from $3 \mathrm{M}$ and the potential for this product to be susceptible to ASR.

Alkali-silica reactivity (ASR) is a chemical reaction that occurs between alkali and reactive silica present in cement and concrete mixes. When this reaction occurs, a gel is formed that absorbs available water and increases internal pressure, eventually causing the cement to fracture. This fracturing renders the cement vulnerable to several hazardous materials that can severally weaken the structure of the cement matrix causing it to fail.

\section{Testing Procedures}

A variation of the Length Change of Hardened Hydraulic-Cement Mortar and Concrete (ASTM C157-93) methods was used to test expansion in the slurries. An expansion measurement is taken at 24 hours, 7 days, 14 days, 28 days and every consecutive month for the duration of the project. For statistical purposes, six replicates of each slurry are made.

A variation of Splitting Tensile Strength of Cylindrical Concrete Specimens (ASTM C496-90) methods were used to determine a change in the tensile strength of specimens as a result of ASR. A tensile strength measurement is performed at 24 hours, 7 days, 28 days, 2 months, 4 months, and 6 months. For statistical purposes, nine replicates of each slurry are made.

\section{Test Slurry Composition}

As a baseline for testing, neat Class $\mathrm{H}$ cement with a density of $16.4 \mathrm{lb} / \mathrm{gal}$ and TXI Lightweight cement with a density of $13.5 \mathrm{lb} / \mathrm{gal}$ will be used. Ultra-light hollow sphere (ULHS) slurries with similar bead concentrations and a density of $9.0 \mathrm{lb} / \mathrm{gal}$ will also be used. Several $0.5 \%$ sodium chloride slurries will be used to evaluate accelerated ASR.

\section{Neat Slurries (Baseline)}

- Class H cement mixed with 4.3 gal of fresh water per sack to achieve a density of $16.4 \mathrm{lb} / \mathrm{gal}$

- TXI Lightweight cement mixed with 6.0 gal of fresh water per sack to achieve a density of $13.5 \mathrm{lb} / \mathrm{gal}$

\section{Ultra-Light Hollow Sphere (ULHS) Slurries}

- Class H cement plus 42.14\% BWOC 3M 6K (6,000 psi) beads mixed with 11.81 gallons of fresh water per sack for a density of $9.0 \mathrm{lb} / \mathrm{gal}$ 
- $\quad$ TXI Lightweight cement plus 37.19\% BWOC 3M 6K (6,000 psi) beads mixed with 12.63 gallons of fresh water per sack for a density of $9.0 \mathrm{lb} / \mathrm{gal}$

\section{Salt Slurries}

- Class $\mathrm{H}$ cement plus $0.5 \%$ BWOC NaCl mixed with 4.3 gallons of fresh water sack for a density of $16.4 \mathrm{lb} / \mathrm{gal}$

- TXI Lightweight cement plus 0.5\% BWOC NaCl mixed with 6.0 gallons of fresh water per sack for a density of $13.5 \mathrm{lb} / \mathrm{gal}$

- Class H cement with 42.14\% BWOC 3M 6K (6,000 psi) beads plus $0.5 \%$ BWOC $\mathrm{NaCl}$ mixed with 11.81 gallons of fresh water per sack for a density of $9.0 \mathrm{lb} / \mathrm{gal}$

- TXI Lightweight cement with $37.19 \%$ BWOC 3M 6K (6,000 psi) beads plus $0.5 \% \mathrm{BWOC} \mathrm{NaCl}$ mixed with 12.63 gallons of fresh water per sack for a density of $9.0 \mathrm{lb} / \mathrm{gal}$

\section{Testing Conditions}

- Application No. $3-128^{\circ} \mathrm{F} / 174^{\circ} \mathrm{F}$ (BHCT/BHST)

- Slurries were tested for a minimum of 200 days.

\section{Mixing Procedures}

Prepare a ULHS slurry as follows.

1. Weigh the appropriate amounts of the cement sample, additives, water, and ULHS into separate containers.

2. Mix the cement slurry according to Appendix A of API RP 10B.

3. Pour the slurry into a metal mixing bowl and slowly add ULHS while continuously mixing by hand with a spatula. Mix thoroughly.

4. Place this slurry in a Waring blender and mix at 4,000 rpm for 15 seconds. Then, return each specimen to lime-saturated water.

\section{Alkali-Silica Reactivity (ASR) Testing for Expansion}

\section{Curing the Specimens}

One of the test slurries (containing six replicates) is prepared per day until all samples for the project have been made. Cure each test specimen in a heated, circulating water bath containing saturated-lime curing water, as described in the procedure below.

1. Remove specimens from the molds at an age of 23 1/2 hours. Age of each specimen is measured from the moment when water is added to the cement during the mixing operation.

- Never strike or jar a specimen during removal.

- Never exert pressure directly against the gage studs.

- Make sure the gage stud holder remains attached to the stud during specimen removal. 
Important-To avoid damaging the specimens during removal from the molds, it may be necessary to leave the specimens in the molds for more than 24 hours. This is especially true for certain slow-hardening cements. If the curing schedule must be extended, make sure that the same curing time is used for all other specimen to be compared, and that all comparison specimens are within $\pm 1 / 2$ hour of the same age at the time the initial comparator reading is performed.

2. Mark specimens for identification or positioning as required with a soft graphite pencil, a graphite liquid that deposits graphite without binder, or waterproof, indelible ink. Never use any other writing instrument.

3. Place the specimens in lime-saturated water maintained at $73.4 \pm \pm^{\circ} \mathrm{F}\left(23.0 \pm 0.5^{\circ} \mathrm{C}\right)$ for a minimum of $15 \mathrm{~min}$. This helps minimize variation in length measurements due to variation in temperature of the specimens.

4. When the specimens are $24 \pm 1 / 2$ hours in age, remove them from water storage one at a time, wipe with a damp cloth, and immediately take a comparator reading. Then, return each specimen in lime-saturated water.

Important-Monitor the curing water weekly to ensure that the lime concentration of the saturated aqueous solution is at $1,600 \mathrm{mg} / \mathrm{L},+/-300 \mathrm{mg} / \mathrm{L}$.

\section{Apparatus}

Molds for cement specimen curing have one section and are constructed as shown in Fig. 6. Molds for test specimens used in determining the length change of cement pastes and mortars produce $1 \times 1 \times 11^{1 / 4}$-in. prisms with a 10 -in. gage length. The gage length is the nominal length between the innermost ends of the gage studs. The parts of the molds should fit tightly and firmly together when assembled, and their surfaces should be smooth and free of pits.

The molds are made of steel not readily attacked by the cement paste, mortar, or concrete. The sides of the molds should be sufficiently rigid to prevent spreading or warping. For the molds shown in Fig. 6, the tolerance on dimension A is \pm 0.03 in. Each end plate of the mold is equipped to hold properly in place during the setting period.

The gage studs are of American Iron and Steel Institute (AISI) 3 Type 316 stainless steel. To prevent restraint of the gage studs before the specimen is removed, the device for holding the gage studs in position is arranged such that it can be partially or completely released after the slurry compacts in the mold. The gage studs are set so their principal axes coincide with the principal axis of the test specimen. For the molds shown in Fig. D1 , gage studs extend into the specimen $0.625 \pm 0.025 \mathrm{in}$. and the distance between the inner ends of the gage studs is $10.00 \pm 0.10 \mathrm{in}$. . Ten inches is the gage length for calculating length change.

\section{Test Measurement}

The comparator for the molds shown in Fig. D-1 features a dial micrometer or other measuring device graduated to read in 0.0001-in. units, accurate within 0.0001 in. in any 0.0010 -in. range, and within 0.0002 in. in any 0.0100 -in. range, and sufficient range (at 
least 0.3 in.) in the measuring device to allow for small variations in the actual length of specimens. The terminals of the comparator are plane, polished, and heat-treated, and are fitted with collars held in place with set screws. The collars extend $0.062 \pm 0.003$ in. beyond the plane face of the terminal and have an inside diameter 0.02 in. greater than the average diameter of the portion of the gage studs that must fit into the collars.

The comparator must allow the checking of the measuring device against a reference bar at regular intervals. The reference bar has an overall length of $115 / 8 \pm 1 / 8$ in. for the specimen in use. The bar is made of a steel alloy with a coefficient of thermal expansion not greater than two millionths per degree Celsius. Each end of the reference bar is fitted with heat-treated, hardened, and polished tips machined to the same shape as the contact end of the gage studs used in test specimens.

\section{Reference Bar}

Place the reference bar (Fig. D-2) in the instrument in the same position each time a comparator reading is taken. Check the dial gage setting of the measuring device by taking a comparator reading of the reference bar at least at the beginning and end of a series of specimen readings to span no more than a half-day, provided the apparatus is kept in a room maintained at constant temperature.

To obtain a comparator reading, perform the following steps.

1. Clean the hole in the base of the comparator into which the gage stud on the lower end of the bar fits.

2. Read and record the comparator indication of the length of the reference bar.

3. Take one bar out of immersion, blot the pins, place the bar in the comparator, read, and record the indication.

4. Return the bar to immersion and clean the hole in the base of the comparator.

5. Repeat the procedure with second and subsequent bars until all bars have been read, returned to immersion, and the readings recorded.

6. After reading the last bar, clean the hole in the comparator base and read and record the reference-bar indication. Blot only around the pins.

Calculate the specimen length change at any age as follows:

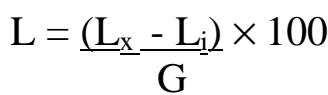

Where:

$L=$ change in length at $x$ age, $\%$

$L x=$ comparator reading of specimen at $x$ age minus comparator reading of reference bar at $x$ age;

$L i=$ initial comparator reading of specimen minus comparator reading of reference bar at that same time

$G=$ nominal gage length, 10 in. 


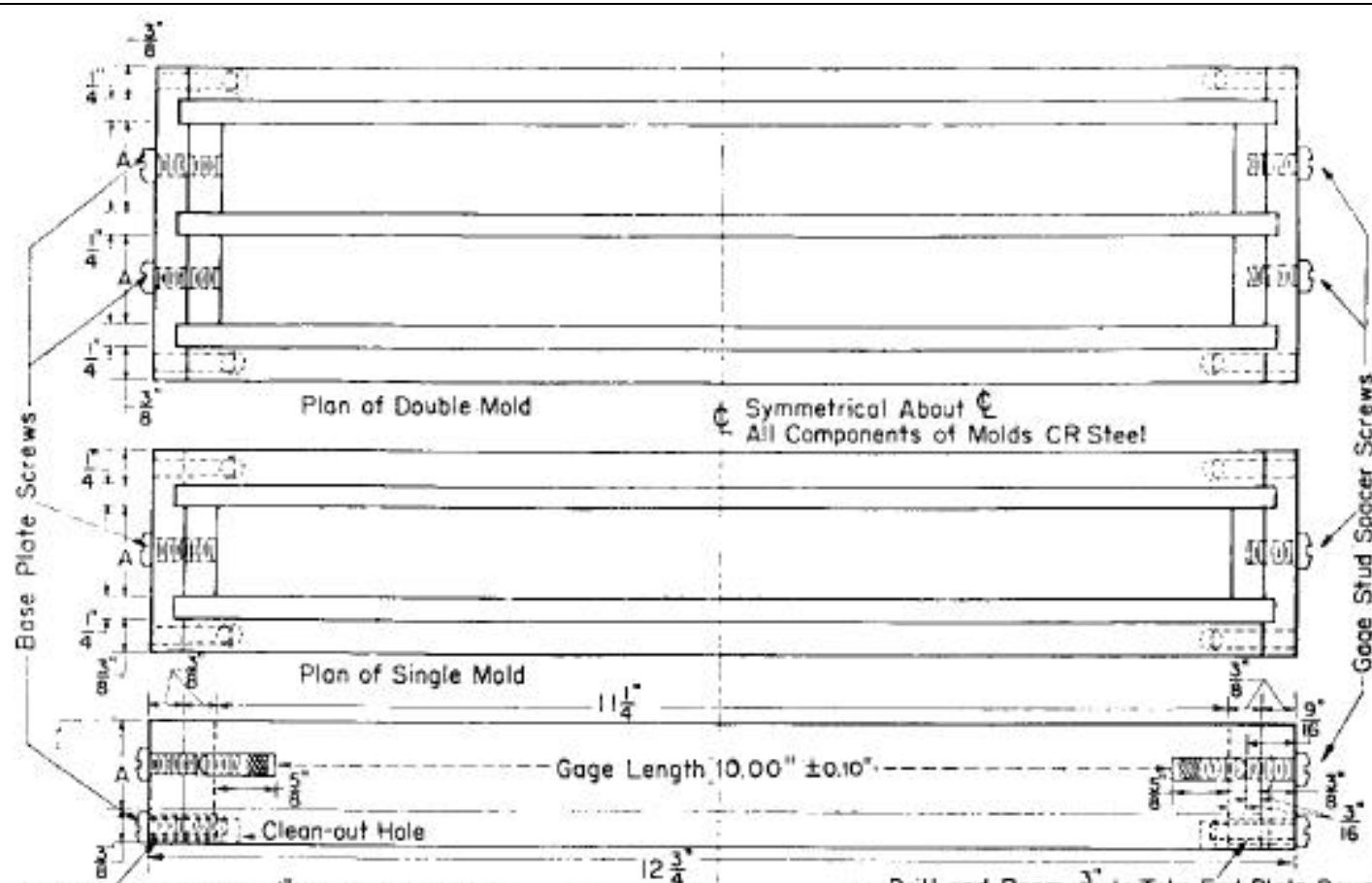

Drill and Tap to Take $\frac{1 \pi}{4}$ Base Plate Screw Elevation of Mold
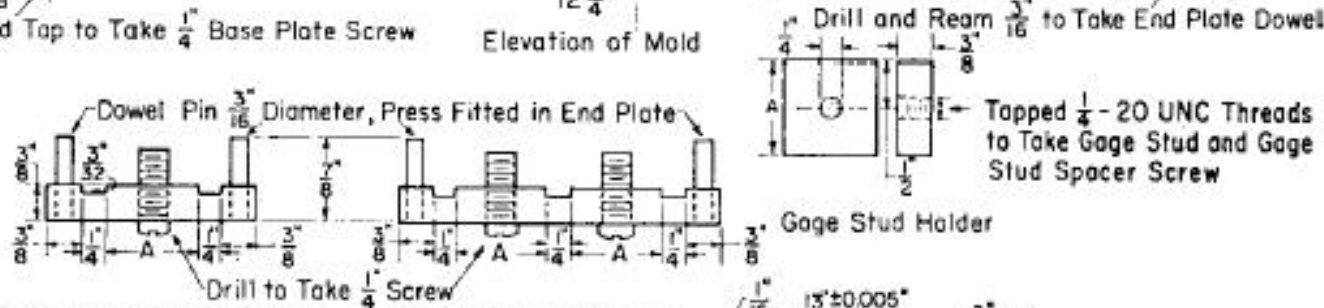

Detoil of End Plate for Single

Mold (Interchangeable)

Detail of End Plate for

3. Gage Stud Holder

to Toke Goge Stud and Goge

$\frac{16}{16} \frac{13}{15}^{2} \pm 0.005^{\circ}$

Double Mold (Interchangeable) $\left.\right|_{-\frac{1}{4}} ^{2}=\frac{3^{*}}{16}:-\frac{5}{16}$ - Pitch diam $0247^{\prime \prime}+0.000^{\circ}$

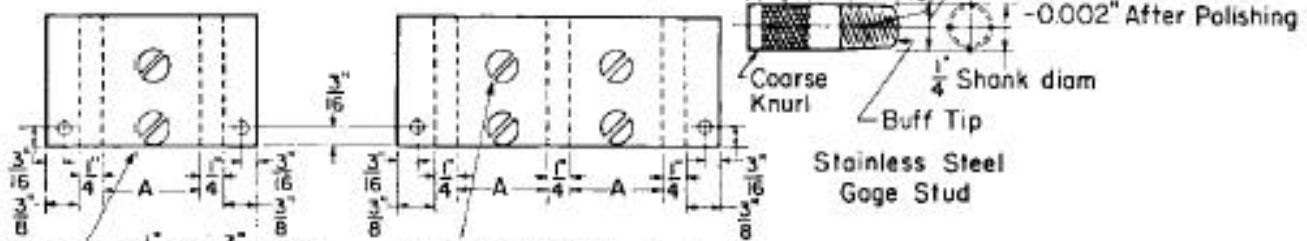

Base Plate Screw $\frac{1^{*}}{4}$ USS $\frac{3^{*}}{4}$ Shaft $\quad \frac{1}{4}^{*}-20$ UNC Fillister Head

$\frac{1}{4} \times \frac{3}{6}$ Round Cop Slotted for Screw Mochine Screw, $\frac{9 " 10}{16}$ long Driver

Elevation of End Plates

Note: Dimension $A$ to be specified by the purchaser.

Figure D-1-Test specimen mold schematics 


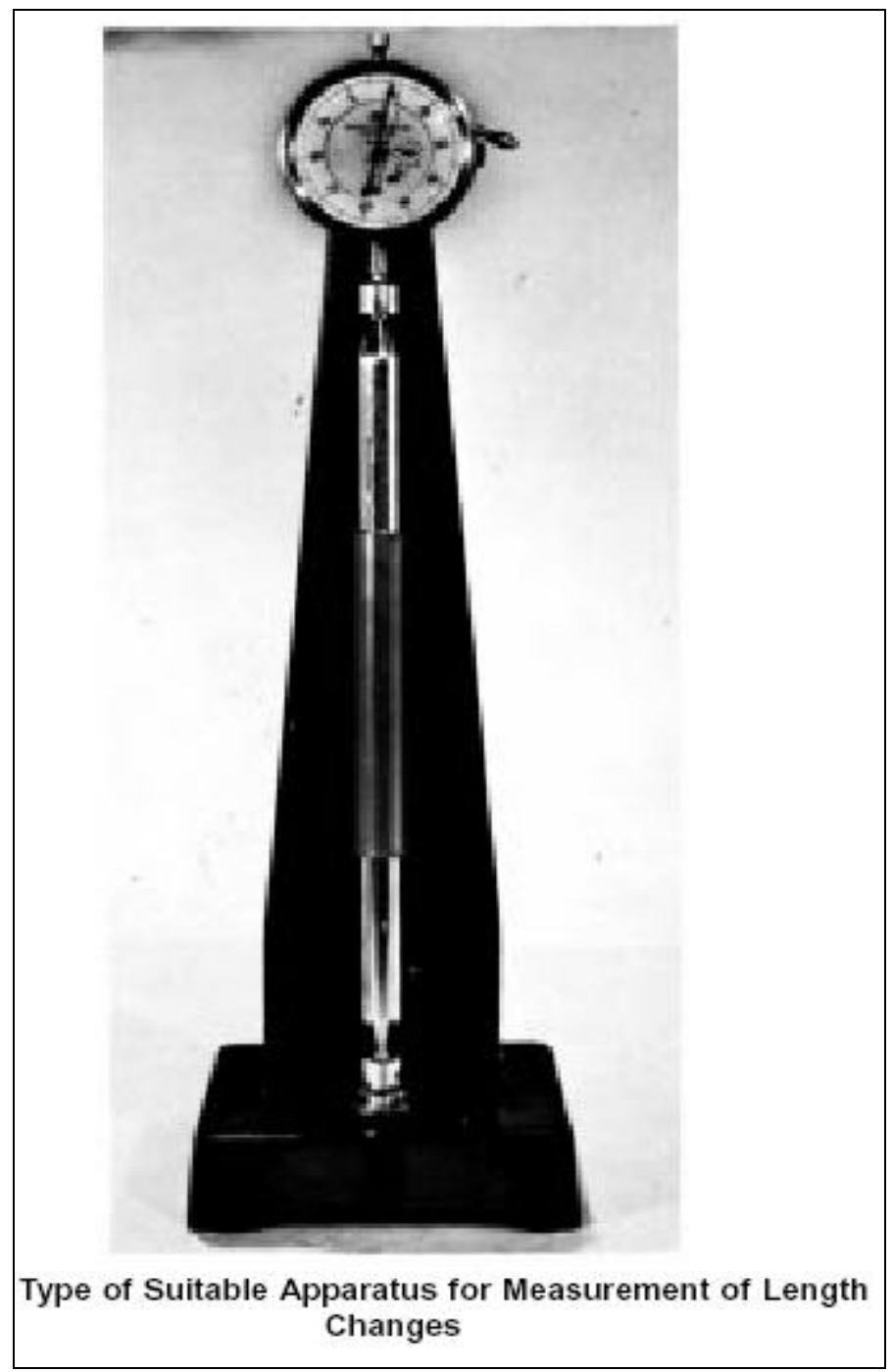

Figure D-2-Reference bar

\section{Alkali-Silica Reactivity (ASR) Testing for Tensile Strength}

The testing method used is similar to that described in ASTM C496-90 (Standard Test Method for Splitting Tensile Strength of Cylindrical Concrete Specimens). For this testing, the slurry is placed in a $1.5 \times 5$-in. mold sealed at both ends.

\section{Curing the Cement Specimens}

One of the test slurries (containing six replicates) is prepared per day until all samples for the project have been made.

Prepare the molds as follows.

1. Place slurry in a mold, filling to approximately one-half of the mold depth, and puddle it.

2. Stir the remaining slurry by hand and place into additional molds and repuddle it. 
3. Seal the molds and place them upright in a heated, circulating water bath at the appropriate curing temperature.

4. Remove specimens from the molds at an age of $231 / 2$ hours. Age of each specimen is measured from the moment when water is added to the cement during the mixing operation.

Important-To avoid damaging the specimen during removal, it may be necessary to leave specimens in the molds for more than 24 hours. This is especially true for certain slow-hardening cements. If the curing schedule must be extended, make sure that the same curing time is used for all other specimen to be compared, and that all comparison specimen are within $\pm 1 / 2$ hour of the same age at the time the initial comparator reading is performed.

\section{Test Measurement}

After curing, the sample is extracted from the mold and cut into 1-in. $\pm 1 / 8$ in. sections in length. The density of each sample is measured before it is measured for tensile strength.

A 1/4-in. section of the top surface of the sample is cut first. Next, the three 1-in.sections to be measured are cut. Each 1-in. $\pm 1 / 8$ in. section is identified as top, middle, and bottom and is measured for tensile strength in the test machine. The remaining sample pieces are discarded. Fig. D-3 shows a general schematic of how each specimen is oriented on its side during testing. The force applied to the specimens is automatically controlled and applied at a constant rate of approximately $0.025 \mathrm{~mm} / \mathrm{hr}$. This testing is carried out at Westport Technology Center.

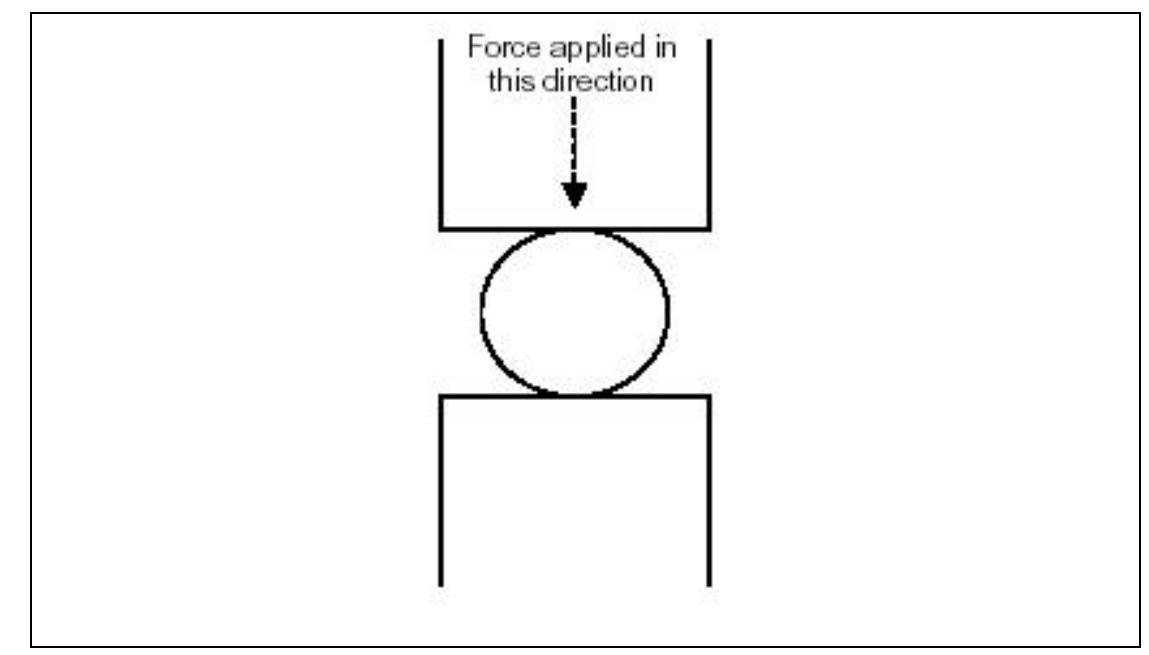

Figure D-3—-Tensile strength Crush Diagram 